\title{
How News and Views are Compiled
}

For the past several years, the section of Nature appearing under the heading News and Views has been concerned principally to provide readers with news of and commentary on developments of interest to professional scientists in all disciplines. One objective has simply been to inform. Although Nature is proud of the frequency with which important discoveries appear first in these pages, there are of course a great many important articles which appear elsewhere which, in the judgment of the staff of Nature, deserve to be brought to the attention of readers, especially those who look to journals like this for a view of how science as a whole is growing. At the same time, it is hoped to provide specialists in a number of important and quickly developing fields of research with an informal guide to the literature which is of most immediate concern to them. But this section has also included comments on articles appearing in Nature or in Nature Physical Science and Nature New Biology.

Most often, the comments on articles which appear in Nature have been designed so as to make the significance of some new development apparent to an audience wider than that of the specialists immediately concerned but also to draw attention, in a way which authors are frequently prevented by their functions from doing, to the probable directions of future developments. The comments on the papers which appear in the Monday and Wednesday editions of the journal are, by contrast, meant as a means by which readers of the principal edition of Nature can learn of important developments due to be announced the following week in the more specialized editions of the journal. It has become clear that these services are valued by many readers and it has therefore been decided that this section of the journal will in future be enlarged and that the corresponding sections of Nature New Biology and Nature Physical Science will be reduced.

In the circumstances, however, it is still more important that readers should understand the way in which this section of the journal is compiled. In the first place, decisions about the developments to be reviewed in News and Views are made by the staff of Nature in consultation with what is now a small army of correspondents dealing with particular fields of science. Inevitably, there will be occasions when these decisions appear arbitrary-no one group of people, however large, could hope to cast a judicial eye over the whole of science and choose accurately and consistently all new developments of importance. The throng of correspondents is itself a varied group, including distinguished scientists with established reputations and much younger people who are, for one reason or another, committed to intensive and systematic reading of the literature in their chosen fields and willing and able to write about new developments in an interesting and even entertaining way. Those who write regularly have in the past been known as "Our Molecular Biology Correspondent" or some other such title, and great care is taken to ensure that there is a $1: 1$ correspondence between these contributors and their titles. In future, such correspondents will be invited to decide for themselves whether their contributions are to be marked with their title or their initials. (This will not apply to the brief summaries of articles due to appear the following week in the Monday and Wednesday editions.) This, it is hoped, will serve to emphasize that these contributions to Nature cannot be and are not intended to be endowed with the majesty of permanent appraisals either of the merit or of the importance of some new development. Their function is frankly journalistic-to inform and, where possible, to stimulate.

An important problem in writing of this kind is the difficulty of being fair. In a necessarily brief comment on some scientific article, where it is necessary to say what the development consists of and possibly to link it with related developments in the field, it may be impossible fully to review the literature from which the new development has sprung and, given the variations there are bound to be in the way in which busy correspondents get their hands on the latest issues of the scientific journals, to take full account of recently published literature which may be relevant.

It is to be hoped that readers will be tolerant of deficiencies of this kind, which would of course be unforgivable in the formal scientific literature but which are permissible within the narrower framework of what is here intended-to inform and sometimes to stimulate. But with the hope of making some articles of this kind more comprehensive as reviews, it is planned that the longer comments on papers considered to be important will in future be tied less closely than in the past to the appearance of an article in Nature, Nature Physical Science or Nature New Biology, and that they will be still longer.

\section{Primute C-Iype Viruses}

Ifl last week's issue of Nature (235, 32; 1972) Spiegelman's group reported molecular hybridization experiments which indicate that human breast cancer cells but not normal breast cells contain RNA molecules with base sequences very similar if not identical to at least part of the RNA genome of mouse mammary tumour virus. The observations of these authors, of course, lend weight to the idea that the RNA virus recently discovered in the milk of some women, which closely resembles in structure and other diagnostic biochemical and biophysical parameters Bittner mouse mammary tumour virus, may be a human breast cancer virus. And if human milk virus is closely related to mouse mammary tumour virus, as seems to be the case, it is not unreasonable to think that human sarcoma and leukaemia viruses, equally closely related to the well characterized mouse leukaemia and sarcoma viruses, probably exist and await detection and isolation.

Indeed Spiegelman and his colleagues, as they mention briefly in their report, have pursued this line of argument and have apparently already shown that samples of several human sarcomas and human leukaemic tissue 